\title{
The "Hitch Stitch": An Effective Method of Preventing Migration in High Tracheal Stenosis
}

\author{
Ravindra M. Mehta ${ }^{a}$ Abhinav Singla ${ }^{a}$ Aashish Shah ${ }^{b}$ Chakravarthi Loknath $^{a}$ \\ a Department of Pulmonary, Critical Care and Sleep Medicine, Apollo Hospitals, and ${ }^{b}$ Department of Gl Oncology \\ Surgery, Minimal Access and Bariatric Surgery, Fortis Hospitals, Bangalore, India
}

\section{Key Words}

Adolescents · Young adults · Middle-aged people .

Bronchoscopy - Tracheal stenosis - Retrospective studies . Silicone stents - Tracheal stenosis - Prosthesis migration · Suture techniques · Foreign-body migration, prevention and control

\begin{abstract}
Background: The incidence of caudal stent migration in high tracheal stenting is $13-21 \%$ and is common with silicone stents. This can lead to major problems, including emergency repeat procedures. Several antimigration methods are described, but have limitations in terms of their success rate, availability, cost or ease of the procedure. Objectives: We describe an innovative method of stent migration prevention using a simple percutaneous anchoring "hitch stitch", validated in a large series. Methods: After tracheal stent placement, an Ethilon suture was passed into the stent lumen through an 18-G needle. To take this suture back to the exterior to complete the stitch, a retrieval loop was passed through another 14-G percutaneous cannula inserted into the stent lumen. Bronchoscopically, using a forceps the first suture was pulled inside the loop, the loop was re-
\end{abstract}

\section{KARGER}

(C) 2016 S. Karger AG, Basel

E-Mail karger@karger.com

www.karger.com/res tracted, the suture was exteriorized, and the knot was completed and embedded subcutaneously. While removing the stent, an endoscopic scissor was used to cut the stitch to free the stent. Results: A total of 42 "hitch stitches" were done in 29 patients over 5 years, predominantly for silicone stents. Indications for stenting included postintubation tracheal stenosis (83.3\%), malignancy (11.9\%) and tracheoesophageal fistula (4.8\%, metal stents). The procedure was successful in $41 / 42(97.6 \%)$ patients. Stitch removal was uncomplicated. Conclusion: This is the largest series of an external stent anchoring procedure as a migration prevention strategy in high tracheal stenting, applicable to both silicone and metal stents. Stent migration prevention using this "hitch stitch" is simple, safe and successful, without any complications during stent removal.

(c) 2016 S. Karger AG, Basel

\section{Introduction}

Tracheobronchial prostheses or airway stents are used to relieve large airway obstruction, in both benign and malignant disease [1]. Complications of tracheal stents include migration, mucus plugging and granulation tis- 
sue formation $[2,3]$. Caudal migration of tracheal stents remains a significant problem, needs repeat procedures, and can be potentially life threatening by causing distal luminal obstruction. The incidence of migration is more frequent in high tracheal stenosis or in any other condition where the stent is in the high to mid-trachea, with no distal support. The incidence of airway stent migration is about $13-21 \%[4,5]$. This is more common for silicone stents than for metal stents, as the self-expanding nature of metal stents promotes better wall adherence $[6,7]$. Strategies to prevent migration in silicone stents include appropriate sizing, the design of stents incorporating studs which get embedded in the airway mucosa, the use of the hourglass stent, and several types of fixation devices [8-13]. These modalities have limitations in terms of their success rate, availability, cost or ease of the procedure, and cosmetic outcome. In this study, we describe an innovative method of stent migration prevention in a large series of patients. Defined as the "hitch stitch," this is an external stent fixation technique using a simple percutaneous anchoring stitch, embedded in the subcutaneous tissue applicable for both silicone and metal stents.

\section{Methods}

The study included 42 consecutive stent stitches placed in 29 patients over 5 years. These patients had silicone or metal stenting for high to mid-tracheal obstruction, and the "hitch stitch" was placed as an antimigration strategy. The "hitch stitch" was done as follows: after stent placement with rigid bronchoscopy and general anesthesia, the stitch site was selected by bronchoscopically observing the indentation caused by an external forceps to confirm placement within the body of the stent - this was usually between the first and second tracheal rings. A small skin and subcutaneous incision (length $6-8 \mathrm{~mm}$ ) was done at the planned stitch site on the anterior aspect of the neck. An 18-G 2-in needle was then inserted percutaneously through one end of this incision to pass through the skin, subcutaneous tissue and through the stent into its lumen, under visual guidance using a flexible bronchoscope passed through the rigid scope. A nonabsorbable Ethilon straight suture was passed through the needle into the stent (Fig. 1a, 2a). The next step was to complete the loop by bringing this suture outside. To take this suture back through the stent lumen to the exterior, a retrieval loop was inserted through another $14-\mathrm{G}$ percutaneous cannula, placed 5-7 $\mathrm{mm}$ away from the first suture, within the incision (Fig. 1b, 2b). Under flexible bronchoscopy guidance, using a flexible forceps, the first straight suture was pulled inside the loop (Fig. 1c, 2c), and by retraction of the loop, the suture was exteriorized (Fig. 1d, 2d), the knot was completed and embedded subcuta-

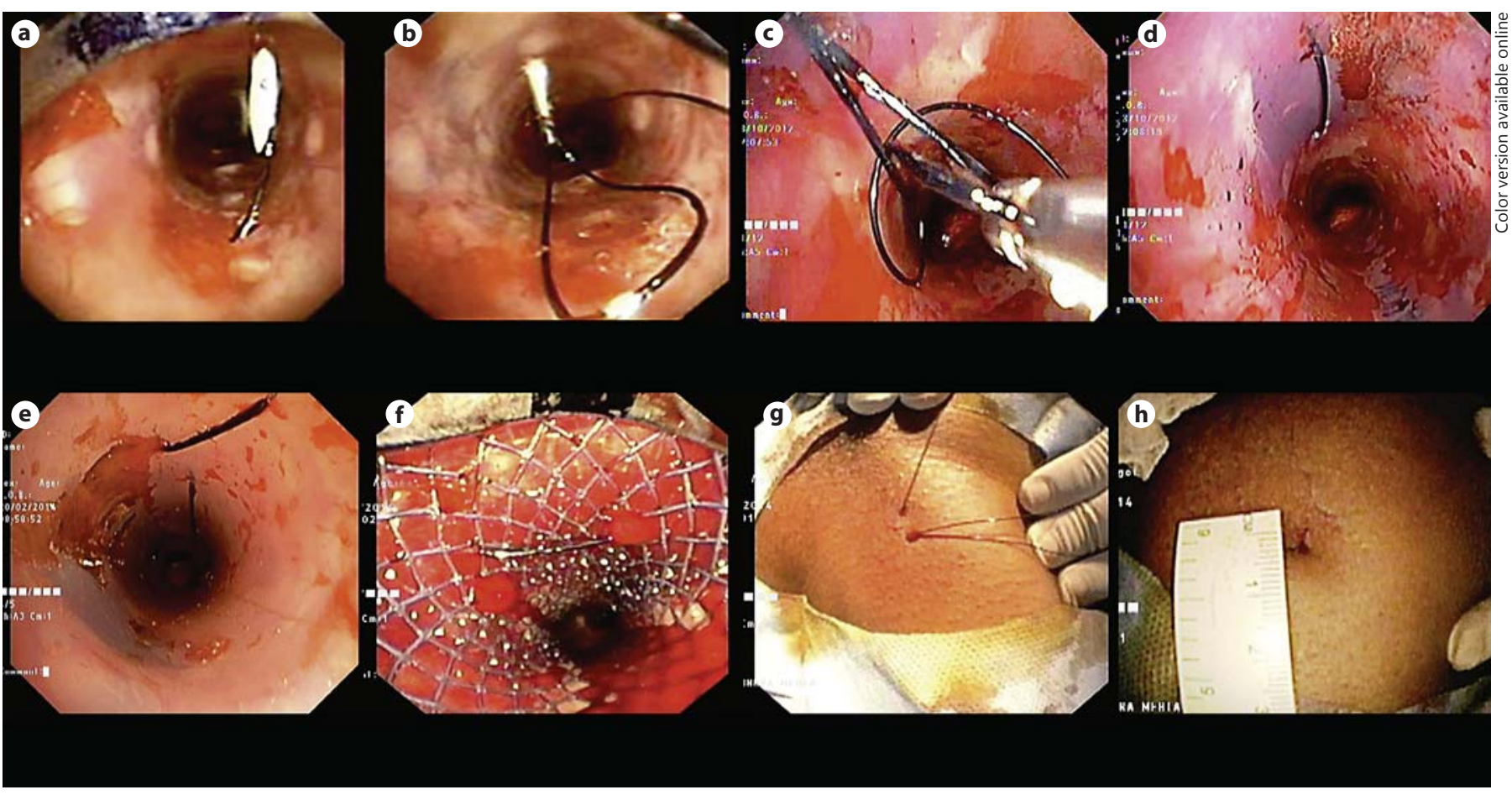

Fig. 1. a Suture threaded through an $18-\mathrm{G}$ needle in the body of stent. b Retrieval loop passed through another catheter. c Suture pulled through retrieval loop. d Stitch completed via pulling loop and knotting subcutaneously. e Extended application of the technique to double-stent stitch. $\mathbf{f}$ Extended application of the technique to SEMS. g External surface showing two ends of suture. $\mathbf{h}$ External surface showing the subcutaneous suture. 


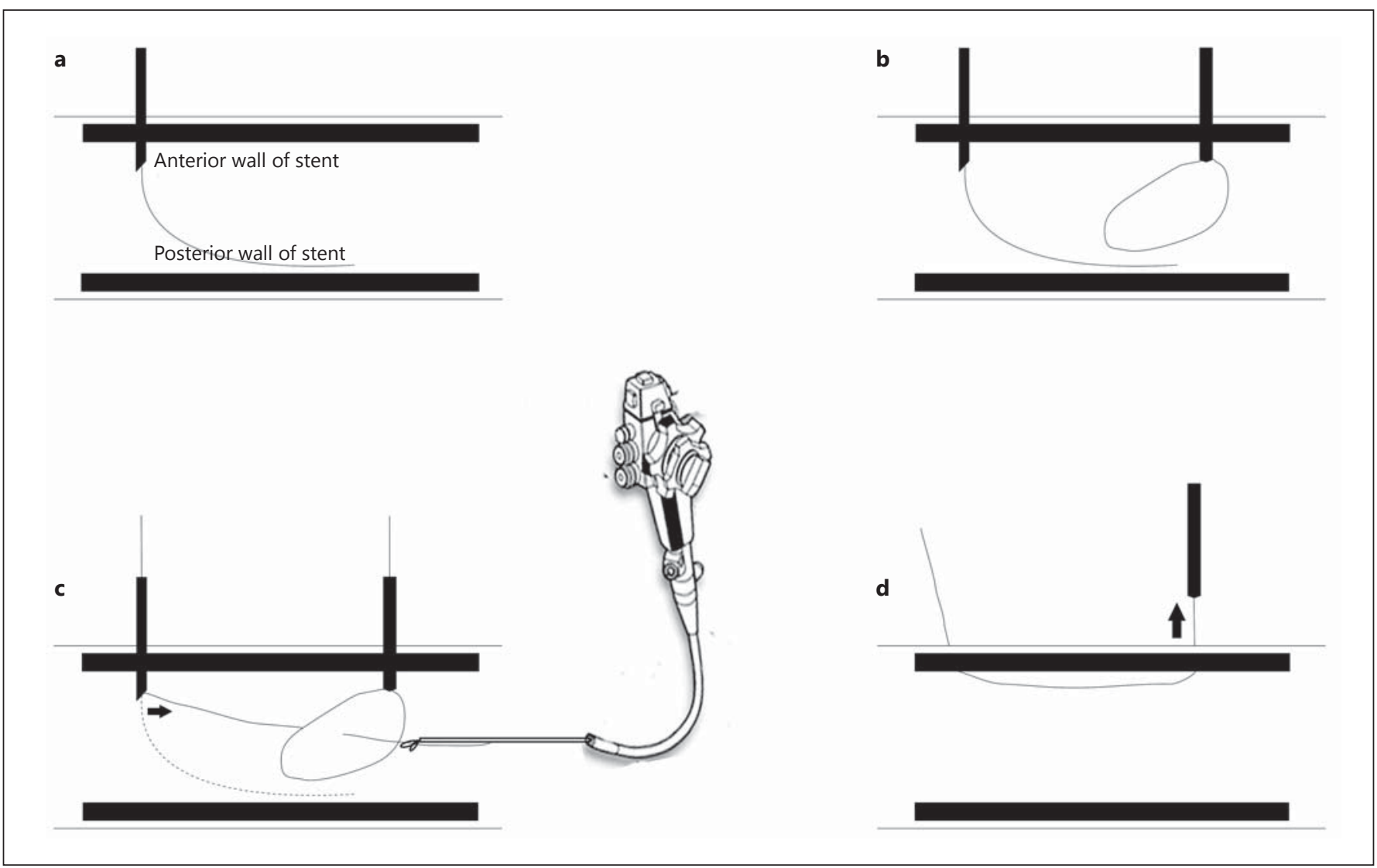

Fig. 2. a Suture passed into the stent lumen through an $18-\mathrm{G}$ needle. b Retrieval loop passed through another 14-G cannula. c Using the bronchoscope and forceps, suture is guided through the retrieval loop. d The retrieval loop is pulled externally to complete the suture.

neously. The entire procedure is also detailed in online supplementary video 1 (see www.karger.com/doi/10.1159/000453075 for all online suppl. material). The overlying skin was sutured, with a residual small insignificant scar. Later, while removing the stent, an endoscopic scissor was used to cut the stitch to free the stent. An institutional review board consent was waived in view of the retrospective nature of the study.

\section{Results}

Over 5 years, 42 stent stitches have been placed in 29 patients (19 males and 10 females). The mean age of the patients was $39.1 \pm 17.5$ years (range: $12-70$ ). Thirty-eight stents were silicone (Dumon stent, Novatech Corporation, La Ciotat, France) and 4 were self-expanding metal stents (SEMS, completely covered nitinol stents, Microtech Corporation). The diagnoses of these patients are summarized in Table 1. The mean length of the stents that were used was $4.7 \pm 1.2 \mathrm{~cm}$ (range: $3.5-7$ ). The diameter of the silicone stents used was $12-16 \mathrm{~mm}$ and that of the metal stents was $16-18 \mathrm{~mm}$, the details of which are presented in Table 2. We also noted the distance of the upper border of the stent from the vocal cord, classified as a distance of $\leq 3 \mathrm{~cm}$ from the vocal cords (36/42) and $>3 \mathrm{~cm}$ from the vocal cords (6/42). In this series, 9/42 "hitch stitches" were placed in prior migration episodes, while $33 / 42$ stitches were placed proactively to mitigate the chances of migration. This decision was based on the high-tracheal location of the stenosis, which is associated with a significant incidence of stent migration as the stenosis dilates, which can lead to emergency procedures in these patients. In the 2 patients with tracheoesophageal fistula, the "hitch stitch" was placed so that the fistula would not get uncovered, in case the stent migrated. The procedure was successful in $41 / 42$ patients (97.6\%), with success defined as absence of migration. In 1 patient, the stent did migrate distally, due to the stitch cutting through - in this patient, the stent was repositioned and a double 
Table 1. Diagnosis of patients

\begin{tabular}{ll}
\hline Diagnosis & $\begin{array}{l}\text { Patients, } n \\
(n=42)\end{array}$ \\
\hline Postintubation tracheal stenosis & 35 \\
Malignancy & 5 \\
Tracheoesophageal fistula & 2 \\
\hline
\end{tabular}

Table 2. Diameter of stents

\begin{tabular}{ll}
\hline $\begin{array}{l}\text { Diameter of the } \\
\text { stents, mms }\end{array}$ & $\begin{array}{l}\text { Patients, } n \\
(n=42)\end{array}$ \\
\hline $\begin{array}{l}\text { Silicone stents } \\
12\end{array}$ & 3 \\
13 & 10 \\
15 & 20 \\
16 & 5 \\
Metal stent & \\
16 & 1 \\
18 & 3 \\
\hline
\end{tabular}

stitch was placed in the vertical and transverse planes to prevent migration. Other minor complications were observed including delayed healing of the skin incision in 2 patients $(4.8 \%)$ and skin infection in 1 patient $(2.4 \%)$, which resolved with oral antibiotics. No other significant symptoms or discomfort were noted. The time taken for placing the "hitch stitch" was approximately 10 min extra after the stent placement was done. The stitches were maintained for the whole duration of the stent placement, ranging from 1 to 54 months, with a mean of $14 \pm 15.4$ months. In 6 patients, the stents were not removed either because of demise due to other causes, or patient reluctance for stent removal due to maintained clinical improvement. The duration of stent placement in this category ranges from 6 to 58 months with a mean of $26 \pm$ 19.5 months. There were no "hitch-stitch"-related complications, even with this long stent duration.

\section{Discussion}

The use of silicone stents for high tracheal stenosis is an established modality, and migration is an important complication of silicone stenting in this location, which limits their effectiveness. Several strategies have been de- scribed to prevent migration in silicone stents. These can be classified as: stent-based strategies, technique-based measures, and external fixation strategies (Table 3).

In the stent-based strategies, the design of the silicone stent itself is configured to promote tracheal wall adherence at several points and prevent migration. The commonly used silicone stent (Dumon stent, Novatech Corporation) incorporates rows of studs on the outer surface, which get embedded in the airway mucosa. One step ahead, another design modification involves the hourglass stent, which has a waist ideally positioned at the area of stenosis, and broader flanges on either side to prevent migration. Variation in the inner area of this stent led to increased mucus retention [12, 13]. Technique-based measures include dilating the stenosis submaximally to maintain a tight grip on the stent, and appropriate sizing using the largest possible stent for the airway to maximize the fit.

Multiple external fixation strategies have been tried over 2 decades to maintain stent position. One such procedure described by Miwa et al. [8] required the use of a conceptually similar fixation apparatus, originally used to fix the stomach to the abdominal wall for gastropexy in endoscopic gastrostomy. In this report, this fixation apparatus was used in 6 patients, predominantly in malignant disease. Closure discomfort of vocal cords was noted as a complication. In addition, the fixation device is not commonly available. Majid et al. [10] also described an external fixation procedure in 9 patients, creating a similar loop, using a special endo-close suturing device with a notched stylet as a suture recovery device to complete the loop. In addition, a silicone button was incorporated on the skin surface as a base to complete the knot. Complications included cellulitis, breakdown of the suture, granulation tissue, and subcutaneous embedding of the external silicone button in 1 patient each. In a similar procedure described in 5 patients by Colt et al. [9], instead of a subcutaneous suture, a propylene button was used for fixation at the entry point in the anterior neck area. The drawback of this technique was discomfort while swallowing due to the movement of the propylene button, and 1 patient had subcutaneous infection. Musani et al. [11], in 7 patients, used a percutaneous endoscopic gastrostomy tube fastener to fix the stent. This procedure, though technically easy, has a metal clamp on the external surface to complete the fixation, with a chance of localized cellulitis, and a need to cover the external portion of the percutaneous endoscopic gastrostomy tube fastener with a waterproof dressing when showering. In addition, if the suture gave way, there was concern for the possibility of 
Table 3. External fixation strategies to prevent stent migration

\begin{tabular}{|c|c|c|}
\hline Method used & $\begin{array}{l}\text { Patients, } \\
n\end{array}$ & Technical issues \\
\hline Technique described by Miwa et al. [8] & 6 & $\begin{array}{l}\text { 1. Special fixation device required } \\
\text { 2. Complications such as discomfort, closure discomfort of vocal cords }\end{array}$ \\
\hline Technique described by Colt et al. [9] & 5 & $\begin{array}{l}\text { 1. Chances of subcutaneous infection through polypropylene button } \\
\text { 2. Swallowing discomfort due to the movement of button }\end{array}$ \\
\hline Technique described by Musani et al. [11] & 7 & $\begin{array}{l}\text { 1. Specialized equipment needed } \\
\text { 2. Metal clamp on the surface of neck } \\
\text { 3. Localized cellulitis and suture breakage }\end{array}$ \\
\hline
\end{tabular}

the T-portion migrating below, with a foreign body in the airway. Another issue to be considered is ease of removal of the stent, when fixation devices are used. Not all of these reports address this issue, with minor difficulties or planning needed in releasing the fixation device in some of these techniques, especially when buttons are used. In contrast, in our series, cutting the stitch with an endoscissor was an easy way to release the stent at the time of removal.

On a practical basis, the stent-based strategies and technical strategies still have a risk of migration due to the dynamic nature of the trachea with an increase in the lumen during inspiration. The shape mismatch between the trachea and the stent is another issue. The smooth tent shape of the trachea with a stiff anterior two-thirds and a floppy posterior wall, in approximation with a round limited flexibility stent itself can lead to migration as the stenosis dilates over time. The external fixation strategies hold greater promise in preventing migration, with the percutaneous nonsurgical methods being easier to implement. Our technique involves one such percutaneous method. The advantages of the "hitch stitch" include safety and efficacy. The stitch was successful in preventing migration in all but 1 patient - this stitch was done early in the learning curve, and we have adapted a semi-tight tensile stitch after that, which prevents the stitch from cutting through. In terms of complexity and time, the equipment needed to complete the stitch is commonly available with no complex tools needed, and the placement of the stitch takes only 10 extra minutes after the stent procedure is done. This can easily be done by the interventional pulmonologist. This approach has mini- mal complications because the suture is covered by a skin cover and free from any foreign bodies, external devices, buttons, or plugs. From the patient point of view, due to the subcutaneous nature of the knot, and a 5- to 7-mm skin scar, it is cosmetically acceptable, and superior to prior techniques which had buttons or plugs requiring extra care. The safety aspect has been mentioned above, with hardly any complications even with a long duration (26 \pm 19.5 months) of the "hitch stitch."

We have extended the use of the "hitch stitch" to SEMS in specific situations. Though SEMS by design and material characteristics have better wall adherence, in conditions such as tracheoesophageal fistula where there is little or no support for the stent, migration can pose a significant problem as it uncovers the fistula, with risk of aspiration pneumonia. In the case of SEMS and tracheoesophageal fistula, the "hitch stitch" plays a pivotal role in preventing migration, which can undermine the entire purpose of stenting. In both our patients with tracheoesophageal fistula and SEMS placed, the location precluded placement of an esophageal stent on the other side. This aspect made this antimigration strategy very important, to maintain the stent position covering the fistula. Apart from 1 patient in the series by Majid et al. [10], our series is the only one to describe external fixation of the metal stents as an effective strategy in such a situation.

A larger question that arises is whether the "hitch stitch" should be used as a primary strategy in conditions such as high tracheal stenosis, or reserved for either operator assessment of fit and potential for migration, or more strictly for stents which migrate. In our series, $21 \%$ were placed for prior migration, and later the threshold 
to place the stitch was low, due to concern of migration and effectiveness of this strategy. This question needs further analysis. The benefit of placing the "hitch stitch" routinely lies in its safety, efficacy, and ease of placement. There is minimal patient discomfort. A major advantage is a reduced need to perform repeat procedures in patients with stent migration. However, an argument against the routine use of this strategy is the extra time needed to place the stitch, infection risk, a small scar, and the need to cut the stitch at the time of removal. In our experience, based on this series, the advantages far outweigh the disadvantages, with hardly any complications. There is a significant advantage of preventing further procedures, especially for patients who reside far away or do not have urgent access to interventional pulmonology referral centers. This has prompted a change of policy from a "rescue" role to a "preventive" consideration at the time of stent placement. In the single patient where the stitch cut through, a "double stitch" was placed which augmented the fixation - this strategy also deserves mention.

Another common cause for stent migration involves endotracheal intubation, which dislodges the stent and can create a ventilatory crisis. Another advantage of the "hitch stitch" is that in stented patients who need endotracheal intubation due to any reason, the chances of dislodging the stent with endotracheal intubation may be drastically reduced.
This is the largest series employing an anchoring stitch when using either a silicone stent or an SEMS for the management of high tracheal stenosis as a stent migration prevention strategy. Stent migration prevention in high tracheal stenosis using such a novel percutaneous stitch is simple, safe, has a high success rate, and reduces the need for repeat procedures. The other major advantage of this procedure is the subcutaneous stitch, with only a small scar on the skin surface. This effective "hitch stitch" has great potential for reducing migration-related complications and preventing recurrent procedures in patients who do not have urgent access to interventional pulmonology centers. Stitch removal at the time of stent removal is also simple, with overall minimal complications of the process. The more provocative question is whether this safe and efficacious strategy should be routinely employed in all high tracheal silicone stents, or reserved for the operator's judgement of migration. In our center, this has become a routine stent migration prevention strategy for all high tracheal stenosis bridged with a silicone stent.

\section{Acknowledgment}

The authors thank Dr. Michael Cutaia, Chief of the Division of Pulmonary and Critical Care, New York Harbour Healthcare System, Brooklyn Campus, for reviewing the paper and providing insights.

\section{References}

1 Saito Y, Imamura H: Airway stenting. Surg Today 2005;35:265-270.

2 Murgu SD, Colt HG: Complications of silicone stent insertion in patients with expiratory central airway collapse. Ann Thorac Surg 2007;84:1870-1877.

3 Dumon J-F, Cavaliere S, Diaz-Jimenez JP, Vergnon J-M, Venuta F, Dumon M-C, Kovitz KL: Seven-year experience with the Dumon prosthesis. J Bronchology Interv Pulmonol 1996;3:6-10.

4 Zakaluzny SA, Lane JD, Mair EA: Complications of tracheobronchial airway stents. Otolaryngol Head Neck Surg 2003;128:478-488.

5 Ferretti G, Kocier M, Calaque O, Arbib F, Righini C, Coulomb M, Pison C: Follow-up after stent insertion in the tracheobronchial tree: role of helical computed tomography in comparison with fiberoptic bronchoscopy. Eur Radiol 2003;13:1172-1178.
6 Zannini P, Melloni G, Chiesa G, Carretta A: Self-expanding stents in the treatment of tracheobronchial obstruction. Chest 1994;106: 86-90.

7 Tsang V: Self-expanding metal stent for tracheobronchial structures. Eur J Cardiothorac Surg 1992;6:555-560.

8 Miwa K, Takamori S, Hayashi A, Fukunaga M, Shirouzu K: Fixation of silicone stents in the subglottic trachea: preventing stent migration using a fixation apparatus. Ann Thorac Surg 2004;78:2188-2190.

9 Colt HG, Harrell J, Neuman TR, Robbins T: External fixation of subglottic tracheal stents. Chest 1994;105:1653-1657.
10 Majid A, Fernandez-Bussy S, Kent M, Folch E, Fernandez L, Cheng G, Gangadharan SP: External fixation of proximal tracheal airway stents: a modified technique. Ann Thorac Surg 2012;93:e167-e169.

11 Musani AI, Jensen K, Mitchell JD, Weyant M, Garces K, Hsia D: Novel use of a percutaneous endoscopic gastrostomy tube fastener for securing silicone tracheal stents in patients with benign proximal airway obstruction. J Bronchology Interv Pulmonol 2012;19:121-125.

12 Bolliger CT, Breitenbuecher A, Brutsche M, Heitz M, Stanzel F: Use of studded Polyflex stents in patients with neoplastic obstructions of the central airways. Respiration 2004;71: 83-87.

13 Vergnon J-M, Costes Fd, Polio J-C: Efficacy and tolerance of a new silicone stent for the treatment of benign tracheal stenosis: preliminary results. Chest 2000;118:422-426. 